\title{
Multicentre analysis of PET SUV using vendor-neutral software: the Japanese Harmonization Technology (J-Hart) study
}

Yuji Tsutsui ${ }^{1}$, Hiromitsu Daisaki ${ }^{2}$, Go Akamatsu ${ }^{3,4}$, Takuro Umeda ${ }^{5}$, Matsuyoshi Ogawa ${ }^{6}$, Hironori Kajiwara ${ }^{7}$, Shigeto Kawase ${ }^{8}$, Minoru Sakurai ${ }^{9}$, Hiroyuki Nishida ${ }^{4}$, Keiichi Magota ${ }^{10}$, Kazuaki Mori ${ }^{11}$, Masayuki Sasaki ${ }^{12^{*}}$ (D) and J-Hart study group ${ }^{12}$

\begin{abstract}
Background: Recent developments in hardware and software for PET technologies have resulted in wide variations in basic performance. Multicentre studies require a standard imaging protocol and SUV harmonization to reduce inter- and intra-scanner variability in the SUV. The Japanese standardised uptake value (SUV) Harmonization Technology (J-Hart) study aimed to determine the applicability of vendor-neutral software on the SUV derived from positron emission tomography (PET) images. The effects of SUV harmonization were evaluated based on the reproducibility of several scanners and the repeatability of an individual scanner.

Images were acquired from 12 PET scanners at nine institutions. PET images were acquired over a period of 30 min from a National Electrical Manufacturers Association (NEMA) International Electrotechnical Commission (IEC) body phantom containing six spheres of different diameters and an ${ }^{18} \mathrm{~F}$ solution with a background activity of $2.65 \mathrm{kBq} /$ $\mathrm{mL}$ and a sphere-to-background ratio of 4 . The images were reconstructed to determine parameters for harmonization and to evaluate reproducibility. PET images with 2-min acquisition $\times 15$ contiguous frames were reconstructed to evaluate repeatability. Various Gaussian filters (GFs) with full-width at half maximum (FWHM) values ranging from 1 to $15 \mathrm{~mm}$ in 1-mm increments were also applied using vendor-neutral software. The SUV $\max$ of spheres was compared with the reference range proposed by the Japanese Society of Nuclear Medicine (JSNM) and the digital reference object (DRO) of the NEMA phantom. The coefficient of variation (CV) of the SUV $\max$ determined using 12 PET scanners $\left(\mathrm{CV}_{\text {repro }}\right)$ was measured to evaluate reproducibility. The CV of the SUV $\max$ determined from 15 frames ( $\left.\mathrm{CV}_{\text {repeat }}\right)$ per PET scanner was measured to determine repeatability.

Results: Three PET scanners did not require an additional GF for harmonization, whereas the other nine required additional FWHM values of GF ranging from 5 to $9 \mathrm{~mm}$. The pre- and post-harmonization $\mathrm{CV}_{\text {repro }}$ of six spheres were (means \pm SD) $9.45 \% \pm 4.69 \%$ (range, 3.83-15.3\%) and 6.05\% $\pm 3.61 \%$ (range, $2.30-10.7 \%$ ), respectively. Harmonization significantly improved reproducibility of PET SUV $\max (P=0.0055)$. The pre- and post-harmonization $\mathrm{CV}_{\text {repeat }}$ of nine scanners were (means $\pm \mathrm{SD}$ ) $6.59 \% \pm 1.29 \%$ (range, 5.00-8.98\%) and 4.88\% $\pm 1.64 \%$ (range, $2.65-6$. $72 \%)$, respectively. Harmonization also significantly improved the repeatability of PET SUV max $(P<0.0001)$.
\end{abstract}

Conclusions: Harmonizing SUV using vendor-neutral software produced SUV $V_{\max }$ for 12 scanners that fell within the

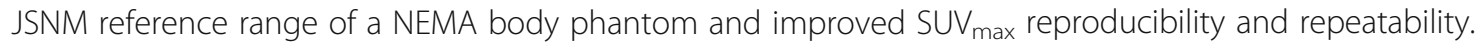

Keywords: FDG PET/CT, SUV, Harmonization, Multicentre study

\footnotetext{
*Correspondence: msasaki@hs.med.kyushu-u.ac.jp

${ }^{12}$ Department of Health Science, Faculty of Medical Sciences, Kyushu

University, 3-1-1 Maidashi, Higashi-ku, Fukuoka 812-8582, Japan

Full list of author information is available at the end of the article
} 


\section{Background}

${ }^{18}$ F-fluoro-2-deoxy-2-D-glucose $\left({ }^{18} \mathrm{~F}\right.$-FDG) positron emission tomography (PET) is a valuable imaging tool for the diagnosis, staging and assessment of the responses of various malignancies to therapy [1-5]. Reports indicate that ${ }^{18}$ F-FDG PET is a more effective biomarker of treatment responses than morphological information because it measures glucose metabolism [6-9]. The standardised uptake value (SUV) has served as a semi-quantitative metric of ${ }^{18}$ F-FDG uptake by tumours.

The SUV must be highly reproducible and repeatable to serve as a reliable biomarker in multicentre trials using various PET scanners, because SUV variability among scanners can contribute to uncertainty in results. The Quantitative Imaging Biomarker Alliance (QIBA), which was organised by the Radiological Society of North America (RSNA), recommended definitions for reproducibility and repeatability $[10,11]$. In short, reproducibility refers to the consistency of values derived from repeated tests of one individual by different operators, different scanners, software, or at different sites and times. Repeatability refers to the consistency of values derived from repeated tests of one individual by a single operator using the same scanner and software. That is, reproducibility refers to inter-scanner variability, whereas repeatability refers to intra-scanner variability.

Recent developments in hardware and software for PET technologies have resulted in wide variations in basic performance. The SUV considerably varies due to biological and technical factors involving the model of the PET scanner, acquisition protocols, reconstruction algorithm and parameters [12-15]. For example, ordered subset expectation maximization (OSEM) with a point spread function (PSF) algorithm improves the spatial resolution, but it causes edge artifacts as overestimation [16, 17]. Therefore, multicentre studies require a standard imaging protocol and SUV harmonization to reduce inter- and intra-scanner variability in the SUV.

Several organizations have published guidelines to standardise ${ }^{18} \mathrm{~F}-\mathrm{FDG}$ PET imaging protocols and harmonize SUV harmonization [14, 18-20]. In terms of SUV harmonization, the European Association of Nuclear Medicine (EANM) and the Japanese Society of Nuclear Medicine (JSNM) have proposed a specified range of recovery coefficients (RC) as a function of sphere size [21]. "Harmonization" is defined herein as a PET image smoothed with an additional Gaussian filter (GF) so that the RC of each sphere falls within the specified range of the body phantom. This harmonization strategy minimises variations in SUV measurements during scan acquisition and processing [22-24] and reduces reconstruction-dependent variability in the PET Response Criteria in Solid Tumours (PERCIST) classification [25].
The usefulness of commercially available software for harmonization was first determined using EQ.PET software [23]. However, a costly, software-specific workstation provided by the Siemens Healthineers is required. The recently commercially available GI-PET software (AZE VirtualPlace Hayabusa, Tokyo, Japan) adjusts the RC of PET images into a reference $\mathrm{RC}$ using an additional GF. This vendor-neutral quantitative software can be installed in general personal computers.

We designed the Japanese SUV Harmonization Technology (J-Hart) study based on this background to determine the applicability of vendor-neutral software to multicentre PET SUV harmonization. We also assessed the effects of SUV harmonization on SUV reproducibility and repeatability.

\section{Methods}

\section{Phantom}

We used a NEMA 2001 International Electrotechnical Commission (IEC) body phantom (Data Spectrum Corp., Durham, NC, USA), consisting of six spheres (Model PET/IEC-BODY/P) of 10, 13, 17, 22, 28 and $37 \mathrm{~mm}$ in diameter, with a wall thickness of $1 \mathrm{~mm}$ in a quasi-cylindrical cavity $(280 \times 210 \times 180 \mathrm{~mm})$. All spheres and the background were filled with solutions containing 10.6 and $2.65 \mathrm{kBq} / \mathrm{mL}$ (at the midpoint of 30 -min acquisitions), respectively, of ${ }^{18}$ F-FDG to obtain a sphere-to-background ratio of 4 .

\section{PET/CT scanners}

Data were acquired at nine Japanese institutions using 12 popular PET systems (Table 1): Discovery ST Elite Performance (DSTEP), Discovery ST Elite (DSTE), Discovery 600 Motion (D600), Discovery 690 (D690) and Discovery IQ (DIQ) scanners (General Electric Medical Systems, Milwaukee, WI, USA); Biograph 64 True Point (BioTP), Biograph mCT 3ring (Bio3R) and Biograph mCT flow 4ring (Bio4R) scanners (Siemens Healthineers, Erlangen, Germany); GEMINI-TF16 (GTF), Gemini GXL (GXL) scanners (Philips Medical Systems, Cleveland, OH, USA); Aquiduo (Aquiduo) and Celesteion PCA-9000A (Celesteion) scanners (Toshiba Medical Systems, Otawara, Japan). The collaborating institutions comprised five university hospitals, three cancer centres and one research institution that normally conduct many oncological PET studies. The dose calibrator and each PET scanner of all evaluated PET/CT systems were cross-calibrated before data acquisition from the phantom according to the guidelines [19].

\section{Region of interest}

The $\mathrm{SUV}_{\max }$ of the six hot spheres was determined from circular regions of interest (ROIs) placed on the 


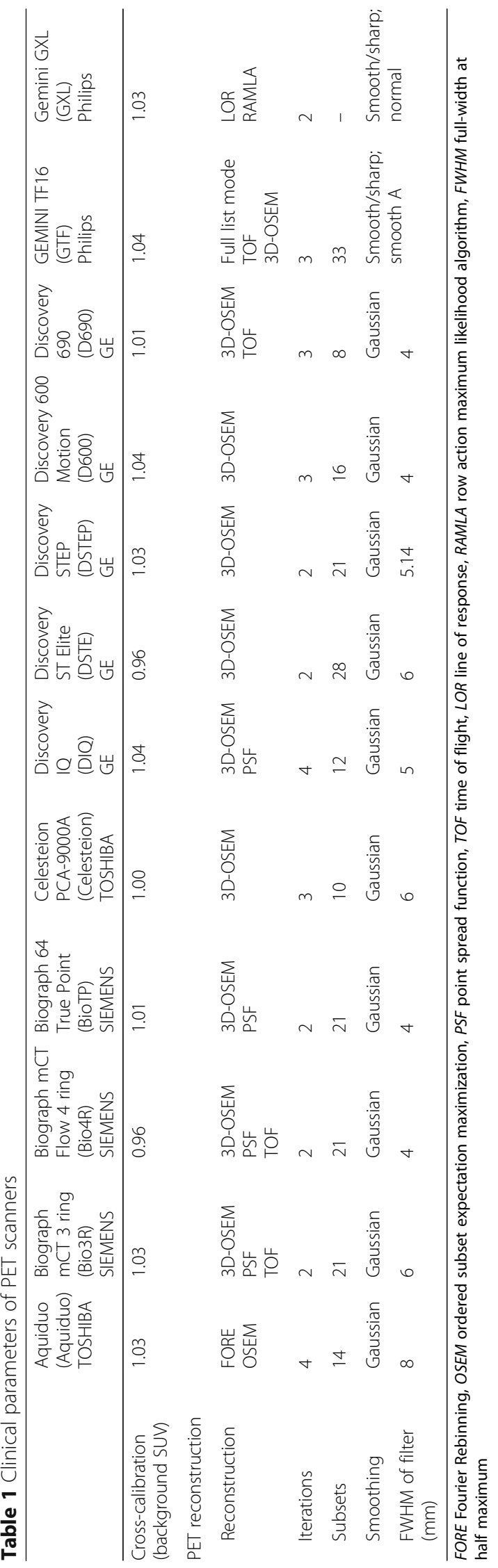


centre slice of images of each sphere. The diameter of each ROI was equal to that of each hot sphere (10, 13, 17, 22, 28 and $37 \mathrm{~mm}$ ). Twelve circular ROIs with a diameter of $10 \mathrm{~mm}$ were placed on the background region at the centre slice of the PET image; the average SUV was calculated from twelve ROIs as the $\mathrm{SUV}_{\text {mean }}$ of the background. The acceptance criterion for the $\mathrm{SUV}_{\text {mean }}$ of the background region was $1.00 \pm$ 0.05 . Table 1 shows the average $\mathrm{SUV}_{\text {mean }}$ of the background region after cross-calibration.

\section{Digital reference object}

The QIBA suggested that computational imaging models or phantom data might play an important role in reproducibility assessment [26] and thus developed an ${ }^{18}$ F-FDG PET/CT digital reference object (DRO), which is a synthetic test object in Digital Imaging and Communications in Medicine (DICOM) format [27]. We obtained the DRO from the QIBA [28]. The mathematically developed DRO of the NEMA body phantom is an ideal object with a uniform background with a transaxial diameter of $20 \mathrm{~cm}$ that simulates a human abdominal cross section with an SUV of 1.00. Six spheres with diameters of 10, 13, 17, 22, 28 and $37 \mathrm{~mm}$ contained an ${ }^{18} \mathrm{~F}$-FDG solution with an SUV of 4.00. The SUV of a central cylinder with a diameter of $5 \mathrm{~cm}$ was 0.00 . A three-dimensional (3-D) GF was applied to the DRO to simulate a PET image with relatively low spatial resolution. The RC of the DRO was included in the JSNM reference range for a GF with a full-width at half maximum (FWHM) value of $10-13 \mathrm{~mm}$, and the RC was highest at a 10-mm FWHM of GF (Fig. 1). We adopted $\mathrm{DRO}_{10 \mathrm{~mm}}$ as the reference SUV $\left(\mathrm{SUV}_{\text {ref }}\right)$ for harmonization.

\section{Data acquisition and processing}

Emission data were acquired from the phantom over a period of $30 \mathrm{~min}$ using list mode with the bed in position 1. A PET image with 30 -min acquisition was reconstructed to determine the parameters for harmonization and to evaluate reproducibility. PET images with 2-min acquisition $\times 15$ contiguous frames were reconstructed to evaluate the repeatability. The reconstruction parameters were individually determined according to the clinical setting at each institution (Table 1). All reconstructed PET images were output in DICOM format.

\section{Harmonization}

We used GI-PET software on a personal computer for harmonization processing. Stand-alone GI-PET software can quantify PET images and it is typically used to adjust spatial resolution to harmonize PET images using a 3-D GF.

The harmonization procedure of GI-PET was as follows: Firstly, reconstructed PET images with 30-min acquisitions in DICOM format were loaded into the GI-PET software. The original voxels were converted into isotropic voxels of an equivalent size in the $x, y$ and $z$ directions using the bi-linear method because the 3D-GF of GI-PET requires isotropic data. A 3-D GF with various FWHM values of $1-15 \mathrm{~mm}$ in $1-\mathrm{mm}$ increments was applied to the PET images, and then, a graph of the $\mathrm{SUV}_{\max }$ of the spheres was generated as a function of the diameter. The FWHM of the GF that provided an $\mathrm{SUV}_{\max }$ within the reference range proposed by the JSNM [21] was determined. The reference ranges of the lower to the upper limits of the SUV $_{\max }$ on 10-, 13-, 17-, 22-, 28- and 37-mm hot spheres were $1.19-2.00,1.52-3.04,2.58-3.71,3.25-$ $4.09,3.56-4.21$ and $3.82-4.17$, respectively. If only one FWHM provided an $\mathrm{SUV}_{\max }$ within the reference range, then that FWHM was taken as the optimal parameter of GF for harmonization. On the other hand, if several FWHM provided $S U V_{\max }$ within the reference range, we compared the root mean square error (RMSE) with $\mathrm{DRO}_{10 \mathrm{~mm}}$. The RMSE is the

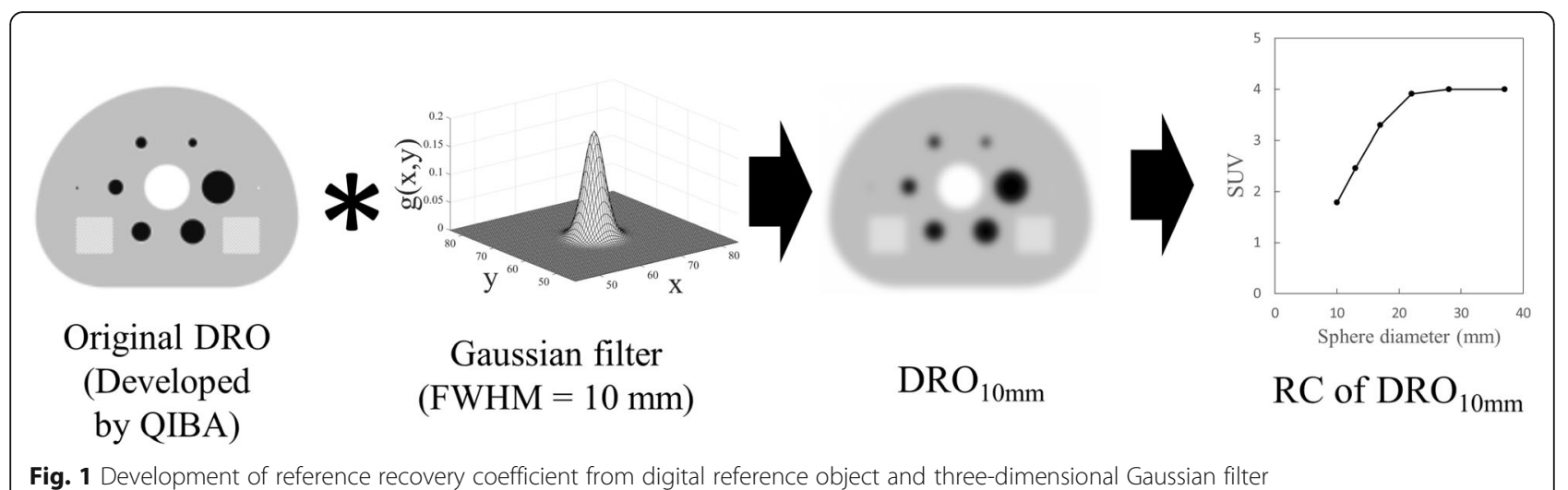

Fig. 1 Development of reference recovery coefficient from digital reference object and three-dimensional Gaussian filter 


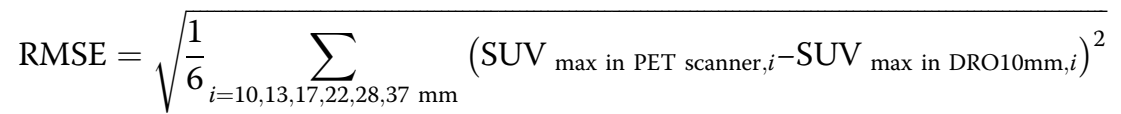

square root of the variance in the $\mathrm{SUV}_{\max }$ of the six spheres between PET images and $\mathrm{DRO}_{10 \mathrm{~mm}}$ and is calculated as:

Finally, we determined the optimal FWHM that provided the smallest RMSE (Fig. 2). RSME is a simple mathematical measure that has been used extensively in nuclear medicine and molecular imaging for several years. Combining RSME with DRO is reportedly useful to create appropriate reconstruction conditions for other types of nuclear medicine image [29].

\section{Reproducibility and repeatability}

We calculated the coefficients of variation $(\mathrm{CV})$ of the $\mathrm{SUV}_{\max }$ on images of spheres with different diameters acquired over a period of $30 \mathrm{~min}$ by 12 PET scanners to evaluate the reproducibility of quantitation and defined the $\mathrm{CV}$ across the scanners as $\mathrm{CV}_{\text {re- }}$ pro. We then compared $\mathrm{CV}_{\text {repro }}$ between the pre- and post-harmonized images calculated as the mean \pm standard deviation (SD). The $\mathrm{CV}$ is the ratio of the standard deviation (SD) of the mean calculated as:

$$
\mathrm{CV}=\mathrm{SD} / \text { mean } \times 100(\%)
$$

We calculated the $\mathrm{CV}$ of $\mathrm{SUV}_{\max }$ on PET images with 2-min acquisitions $\times$ contiguous 15 frames for each PET scanner and each sphere size to evaluate the repeatability of quantitation and then defined the $\mathrm{CV}$ across the frames as $\mathrm{CV}_{\text {repeat }}$. We then compared $\mathrm{CV}_{\text {repeat }}$ between the pre- and post-harmonized images calculated as the mean $\pm \mathrm{SD}$.

\section{Statistical analyses}

Differences in $\mathrm{CV}_{\text {repro }}$ and $\mathrm{CV}_{\text {repeat }}$ were compared among six and 54 pairs, respectively, between preand post-harmonization by Wilcoxon signed-rank tests using JMP $^{\circ} 13$ (SAS Institute Inc., Cary, NC, USA). $P$ values $<0.05$ were considered statistically significant.

\section{Results}

\section{Harmonization}

The SUV $V_{\max }$ fell within the JSNM reference range in three PET scanners (DSTE, DSTEP and GXL) without adding a GF (Table 2). Thus, adjustment was not required for harmonization. The FWHM values of the additional GF that resulted in an $\mathrm{SUV}_{\max }$ within the JSNM reference range were 5-7, 5-9, 8-10, 9, 6-10, 8-9, 6-9, 6-9 and 5-7 $\mathrm{mm}$ for the Aquiduo, Bio3R, Bio4R, BioTP, Celesteion, DIQ, D600, D690 and GTF, respectively. The RMSE compared with $\mathrm{DRO}_{10 \mathrm{~mm}}$ was the smallest at FWHM values of 5,5 , $8,9,6,8,6,6$ and $5 \mathrm{~mm}$, respectively (Table 2). The $\mathrm{SUV}_{\max }$ of these nine scanners fell within the JSNM reference range only after harmonization. Figure 3 shows the pre- and post-harmonization RC of 12 PET scanners.

\section{Reproducibility}

Figure 4 shows the pre- and post-harmonization $\mathrm{CV}_{\text {repro }}$ of the 30-min PET images among 12 PET scanners. The (means $\pm \mathrm{SD}$ ) pre- and post-harmonization $\mathrm{CV}_{\text {repro }}$ of six spheres were $9.45 \% \pm 4.69 \%$ (range, $3.83-15.3 \%$ ) and $6.05 \% \pm 3.61 \% \quad$ (range, $2.30-10.7 \%$ ), respectively. Harmonization significantly improved the reproducibility of the PET SUV $\mathrm{max}_{\max }(P=0.0055)$.

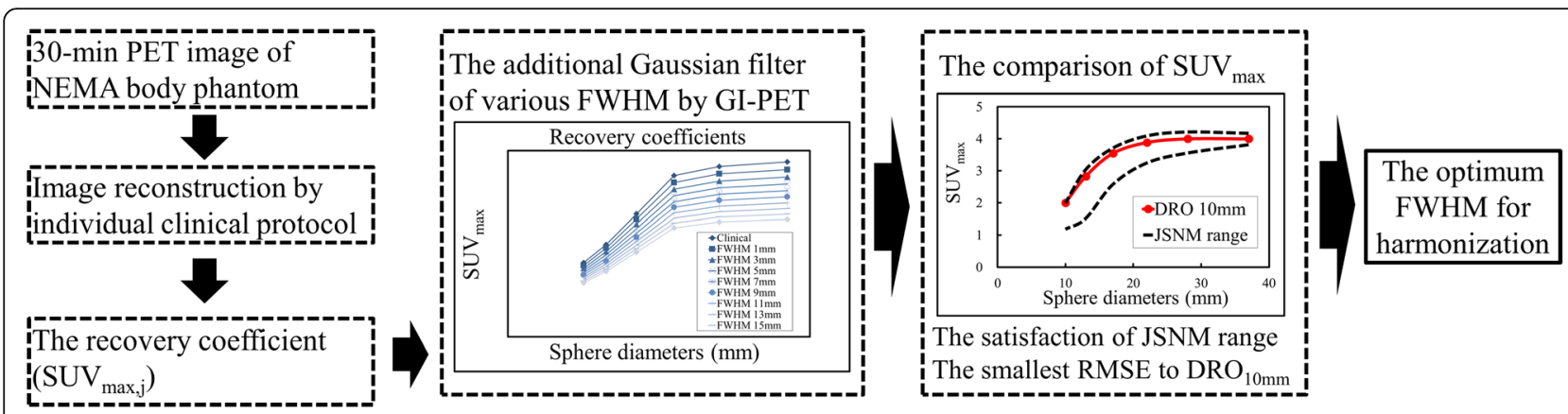

Fig. 2 Flow chart used to determine the optimal FWHM of additional Gaussian filter for harmonization 
Table 2 Full-width at half maximum for SUV harmonization of PET scanners

\begin{tabular}{lll}
\hline PET scanner & $\begin{array}{l}\text { Range of FWHM for GF } \\
\text { for inclusion in JSNM } \\
\text { reference range }(\mathrm{mm})\end{array}$ & $\begin{array}{l}\text { Optimum FWHM for GF } \\
\text { to obtain smallest RMSE } \\
\text { compared with } \\
\text { DRO }_{10 \mathrm{~mm}}(\mathrm{~mm})\end{array}$ \\
\hline Aquiduo & $5-7$ & 5 \\
Bio3R & $5-9$ & 5 \\
Bio4R & $8-10$ & 8 \\
BioTP & 9 & 9 \\
Celesteion & $6-10$ & 6 \\
DIQ & 8,9 & 8 \\
DSTE & No filter & No filter \\
DSTEP & No filter & No filter \\
D600 & $6-9$ & 6 \\
D690 & $6-9$ & 6 \\
GTF & $5-7$ & 5 \\
GXL & No filter & No filter \\
\hline
\end{tabular}

DRO digital reference object, FWHM full-width at half maximum, GF Gaussian filter, RMSE root mean square error

\section{Repeatability}

Figure 5 shows the $\mathrm{CV}_{\text {repeat }}$ of images across 15 frames acquired from each PET scanner. The post-harmonization $\mathrm{CV}_{\text {repeat }}$ of the nine PET scanners that required an additional GF for harmonization (Aquiduo, Bio3R, Bio4R, BioTP, Celesteion, DIQ, D600, D690 and GTF) was lower than that at pre-harmonization (Table 3). The pre- and post-harmonization $\mathrm{CV}_{\text {repeat }}$ (means $\pm \mathrm{SD}$ ) of nine scanners were $6.59 \% \pm 1.29 \%$ (range, $5.00-8.98 \%$ ) and $4.88 \% \pm 1.64 \%$ (range, 2.65$6.72 \%)$, respectively. Harmonization significantly improved the repeatability of the PET $\operatorname{SUV}_{\max }(P<0.0001)$.

\section{Discussion}

The J-Hart study examined whether a vendor-neutral software could harmonize SUV across 12 PET scanners from various manufacturers. We obtained an appropriate FWHM of GF for harmonization by comparing the $\mathrm{SUV}_{\max }$ of 12 PET scanners at nine institutions with both the JSNM reference range and the $\mathrm{DRO}_{10 \mathrm{~mm}}$. Harmonization significantly improved the reproducibility and repeatability of the $\mathrm{SUV}_{\max }$.

Our harmonization process was based on the simple down-smoothing method proposed by Boellaard et al. [30]. This strategy has been widely applied to SUV harmonization $[15,31]$ and provides a pair of PET images with relatively high and low resolution. With a comparable SUV across different scanners as well as in EQ.PET software, not only high-resolution images for lesion detection but also harmonized images can be obtained [23]. The EANM provides the strategy for harmonization applied to RC [19], whereas JSNM recommends harmonization applied to $\mathrm{SUV}_{\max }$ that can detect errors such as cross-calibration. Therefore, $\mathrm{SUV}_{\max }$ data are presented in graphs of the present study.

Quak et al. validated a proprietary software tool (EQ.PET) to harmonize SUV across three PET systems independently of the applied reconstruction algorithm [23]. They reported that all RC of $\mathrm{SUV}_{\max }$ fell within EANM curve after applying the EQ.PET filter. The present study confirmed that vendor-neutral GI-PET software could harmonize the SUV $_{\text {max }}$ across 12 PET scanners. As a result, all SUV$\max$ fell within in the JSNM reference range on GI-PET after adding a GF. Therefore, we consider that GI-PET and EQ.PET are equally useful for
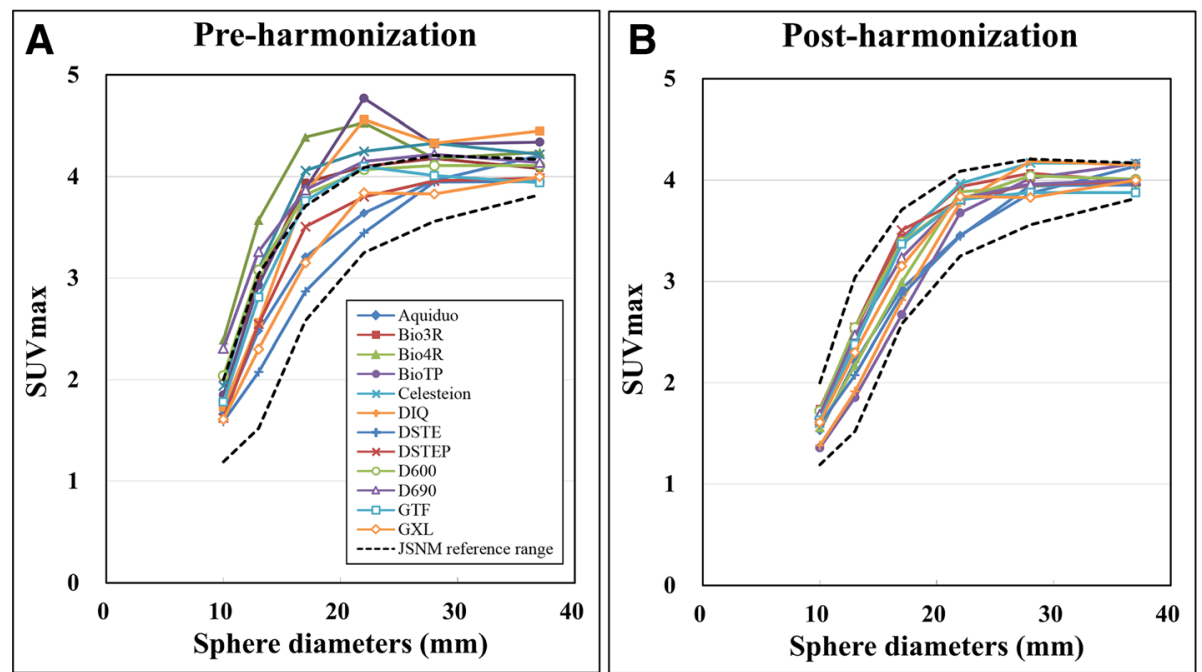

Fig. 3 Recovery coefficients of 12 PET scanners obtained from 30-min PET image. Comparison among a pre- and $\mathbf{b}$ post-harmonization RC 


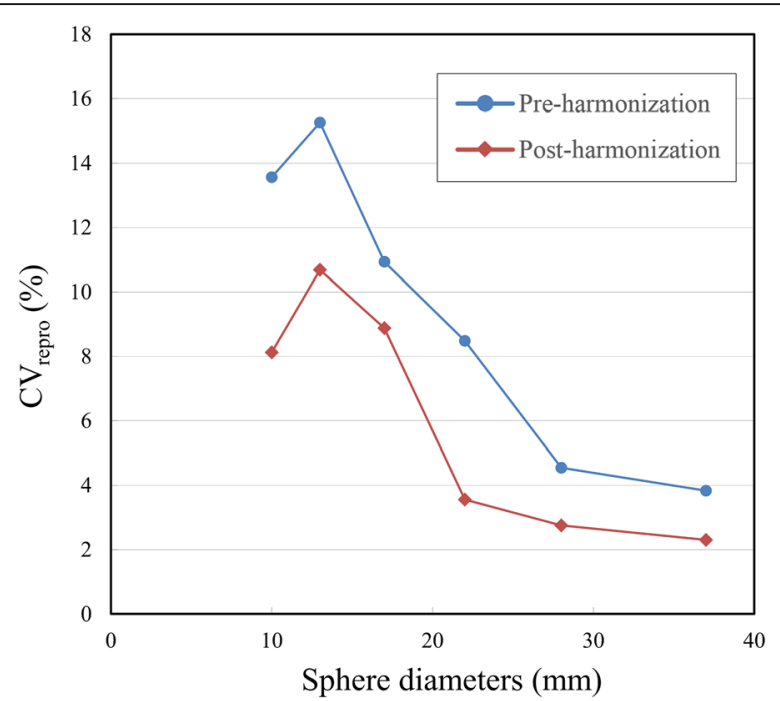

Fig. 4 Effects of harmonization on reproducibility. Pre- and postharmonization comparisons of coefficients of variation of SUV $V_{\max }$ for 12 PET scanners ( $\left.C V_{\text {repro }}\right)$

harmonization. The post-harmonization $\mathrm{SUV}_{\max }$ of various PET scanners became comparable based on the same standard, which is relevant to multicentre and follow-up studies that use various PET scanners. E Q.PET is a proprietary software that can only be installed on its own dedicated workstation with PET system. In contrast, GI-PET is a vendor-neutral software that can be applied at any institution using only a personal computer, regardless of the PET system. Although the present study used the JSNM reference range of $\mathrm{SUV}_{\max }$, the EANM reference range can be used in vendor-neutral software.

Harmonization of the SUV improved the $\mathrm{CV}_{\text {repro of }}$ SUV $_{\max }$ across 12 scanners in the present study. Takahashi et al. reported a maximum difference of $45.7 \%$ in the $S U V_{\max }$ with a $27-\mathrm{mm}$ sphere across five different PET scanners [32]. Such a substantial variation is a problem for reproducibility. Quak et al. evaluated harmonization across three PET scanners using EQ.PET for 1380 FDG-avid tumours [23]. They reported that the mean ratio with and without PSF of the $\mathrm{SUV}_{\text {max }}$ for 1380 tumour lesions was 1.46 (95\% CI 0.86-2.06) before and 1.02 (95\%CI 0.88-1.16) after harmonization. Because the mean value, $+1.96 \times \mathrm{SD}$, was the upper limit of the $\mathrm{CI}$, the SD before harmonization was 0.31 , thus yielding a CV of $21 \%$. The SD after harmonization was 0.07 , thus yielding a $\mathrm{CV}$ of $7 \%$. The present study found that GI-PET im-

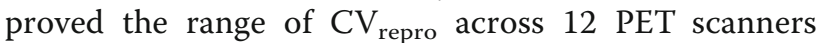
from $3.83-15.3 \%$ to $2.30-10.7 \%$, suggesting that inter-scanner variability was improved equally well by GI-PET and EQ.PET. Harmonization is thought to decrease differences in variations among acquisition protocols (2D, 3D, TOF, etc.), machine specificities (such as type of crystal) and other elements (clock synchronisation, activity preparation and injection). However, their study included 517 patients with tumours who were examined using three PET scanners, whereas we examined a NEMA IEC body phantom using 12 PET scanners. The difference may also be affected by the biological features of tumours and patients. Patients should ideally be clinically assessed using the same scanner. However, patients often undergo PET evaluations with different scanners within the same institution. Multicentre studies usually have different scanners, and thus, therapeutic effects can be assessed using various scanners, and patients are often transferred to other hospitals for advanced specialist care.

Harmonization of the SUV also improved the $\mathrm{CV}_{\text {re- }}$ peat of $\mathrm{SUV}_{\text {max }}$ across 15 frames in each scanner. Velasquez et al. evaluated SUV repeatability in a study of ${ }^{18}$ F-FDG PET and found that the intra-subject $\mathrm{CV}$ for $\mathrm{SUV}_{\max }$ was $10.7 \%$ [33]. Doot et al. evaluated the influence of the FWHM of a GF on the repeatability of phantom data and found that increase of smoothing parameter decreased the standard deviation (SD) of the RC [34]. Kelly et al. evaluated the effects of harmonization on quantitative variation in the $\mathrm{SUV}_{\max }$ relative to the reconstruction protocol. They found that the CV of the $\mathrm{SUV}_{\max }$ across 15 repeat scans pre-harmonization was $2.81 \%, 3.25 \%$ and $4.69 \%$ for OSEM, OSEM+PSF and OSEM+PSF+ time of flight (TOF), respectively, whereas those values after harmonization were $2.28 \%, 2.00 \%$ and $2.58 \%$, respectively [31]. Harmonization is considered to improve the repeatability of any reconstruction protocol.

The $\mathrm{CV}_{\text {repeat }}$ of all scanners and spheres was $<10 \%$ except for that with the $10-\mathrm{mm}$ sphere using the DSTE. With respect to CV repeatability, Lodge et al. reported that the within-subject CV of a tumour SUV was $10 \%$ when acquired with careful attention to the protocol [35]. Data acquisition with the DSTE for clinical examinations requires 3 min per bed position, whereas our phantom study acquired data for $2 \mathrm{~min}$ per frame in the evaluation of repeatability. This might be why the $\mathrm{CV}_{\text {repeat }}$ of the $10-\mathrm{mm}$ sphere using DSTE was $>10 \%$.

The SUV $_{\max }$ of all PET scanners in the present study was above the lower limit of the reference SUV range. Lasnon et al. examined the $\mathrm{RC}$ using Biograph TrueV and found that the $\mathrm{RC}$ for a $10-\mathrm{mm}$ sphere on OSEM images was slightly below the lower limit of the EANM proposed range [15]. Harmonization using a GF is available when the $\mathrm{RC}$ is above the lower limit of the reference range, and it cannot proceed when the $\mathrm{RC}$ is below this limit. However, since some 


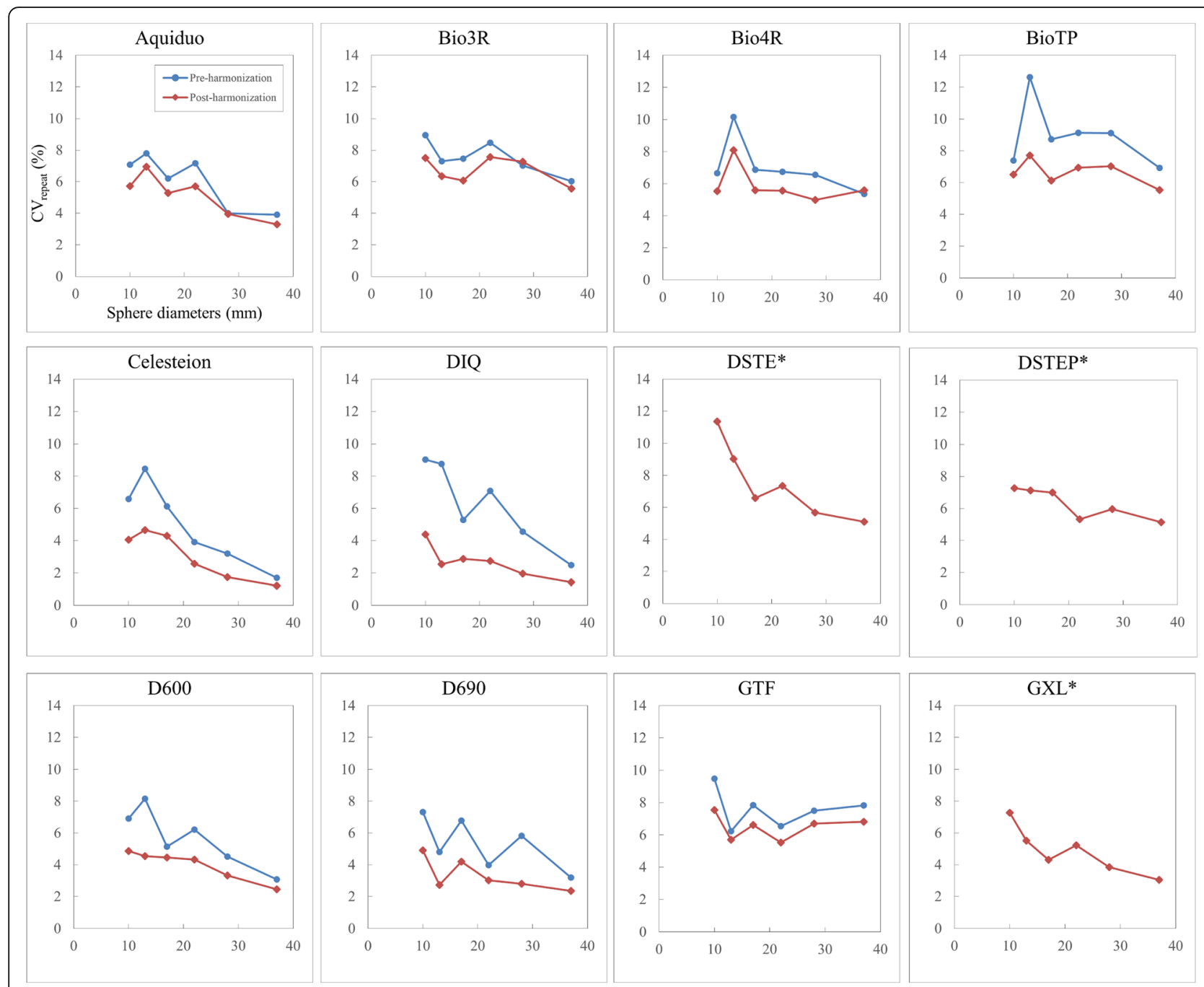

Fig. 5 Effects of harmonization on repeatability. Pre- and post-harmonization comparisons of coefficients of variation of the SUV $\max$ for 15 frames ( $\left(V_{\text {repeat }}\right)$. The asterisk symbol indicates three PET scanners (DSTE, DSTEP and GXL) did not require additional Gaussian filter for harmonization

older PET and PET/CT findings of low spatial resolution might have an $\mathrm{SUV}_{\max }$ below the reference range, the reconstruction settings (including reconstruction methods, matrix, pixel size, iterations, subsets and post-filter) should be carefully considered to obtain an RC within the reference range.

Several limitations are associated with the present study. The basic performance of PET/CT has improved in recent years as the technology has progressed. Therefore, the reference range of the SUV might change with future advancements in PET/CT. We only assessed the $\mathrm{SUV}_{\max }$, although other metrics, such as metabolic tumour volume and total lesion glycolysis, are also useful [36]. The reproducibility and repeatability of new metrics that might be reliable biomarkers should be evaluated. Differences in the voxel size depending on the reconstruction protocol require consideration. GI-PET automatically converts the original voxel size to the isotropic voxel size, which might result in the SUV differing from the value obtained on a proprietary workstation. A vendor-neutral software for SUV harmonization should be able to apply a 3D-GF to original (non-isotropic voxel) data. The present study is evaluated using only the phantom. Therefore, the feasibility of vendor-neutral software in clinical practice requires validation by further clinical studies.

\section{Conclusions}

We harmonized quantitative values among 12 PET scanners using a commercial vendor-neutral software. This harmonization strategy based on the simple down-smoothing method improved the reproducibility and the repeatability of the SUV determined from a 
Table 3 Pre- and post-harmonization coefficients of variation across 15 frames of 12 PET scanners

\begin{tabular}{|c|c|c|}
\hline & $\mathrm{CV}_{\text {repeat }}(\%)$ & \\
\hline & Pre-harmonization & Post-harmonization \\
\hline Aquiduo & $6.03 \pm 1.54$ & $5.15 \pm 1.21$ \\
\hline Bio3R & $7.54 \pm 0.95$ & $6.72 \pm 0.77$ \\
\hline Bio4R & $7.06 \pm 1.48$ & $5.89 \pm 1.01$ \\
\hline BioTP & $8.98 \pm 1.83$ & $6.64 \pm 0.69$ \\
\hline Celesteion & $5.00 \pm 2.28$ & $3.09 \pm 1.32$ \\
\hline DIQ & $6.20 \pm 2.33$ & $2.65 \pm 0.92$ \\
\hline DSTE & $7.51 \pm 2.13$ & - \\
\hline DSTEP & $6.30 \pm 0.87$ & - \\
\hline D600 & $5.66 \pm 1.65$ & $3.99 \pm 0.84$ \\
\hline D690 & $5.31 \pm 1.47$ & $3.33 \pm 0.90$ \\
\hline GTF & $7.57 \pm 1.06$ & $6.48 \pm 0.69$ \\
\hline GXL & $4.86 \pm 1.35$ & - \\
\hline
\end{tabular}

CV coefficient of variation

hot lesion. Therefore, this software might enable comparison of SUV directly across different scanners and facilitate multicentre oncology PET studies.

\section{Abbreviations}

CV: Coefficient of variation; DICOM: Digital Imaging and Communications in Medicine; DRO: Digital reference object; FDG: Fluorodeoxyglucose; FWHM: Full-width at half maximum; GF: Gaussian filter; IEC: International Electrotechnical Commission; J-Hart study: Japanese SUV Harmonization Technology study; JSNM: Japanese Society of Nuclear Medicine; NEMA: National Electrical Manufacturers Association; OSEM: Ordered subset expectation maximization; PERCIST: PET Response Criteria in Solid Tumours: PET/CT: Positron emission tomography/computed tomography; PSF: Point spread function; QIBA: Quantitative Imaging Biomarker Alliance; RC: Recovery coefficients; RMSE: Root mean square error; ROI: Region of interest; RSNA: Radiological Society of North America; SD: Standard deviation; SUV: Standardised uptake value; TOF: Time of flight

\section{Acknowledgements}

The authors thank the staff of Kyushu University Hospital, National Institute of Radiological Sciences, Cancer Institute Hospital, Yokohama City University Hospital, National Center for Global Health and Medicine, Kyoto University Hospital, Clinical Imaging Center for Healthcare, Nippon Medical School, Institute of Biomedical Research and Innovation, Hokkaido University Hospital and Toranomon Hospital for their valuable support.

\section{Availability of data and materials}

Please contact the author for data requests.

\section{Authors' contributions}

MS is the project leader and corresponding author. GA and HN (DSTEP D690), HK (D600), KMA (Bio4R), KMO (BioTP, GXL), MS (GTF), MO (Celesteion), SK (DIQ), TU (Aquiduo) and YT (Bio3R and DSTE) carried out the data acquisition. HD and YT participated in the data analysis. The J-Hart study group conceived of the study concept and design. All authors read and approved the final manuscript.

\section{Ethics approval}

Not applicable

\section{Consent for publication}

Not applicable.

\section{Competing interests}

The authors declare that they have no competing interests.

\section{Publisher's Note}

Springer Nature remains neutral with regard to jurisdictional claims in published maps and institutional affiliations.

\section{Author details}

'Division of Radiology, Department of Medical Technology, Kyushu University Hospital, 3-1-1 Maidashi, Higashi-ku, Fukuoka 812-8582, Japan. ${ }^{2}$ Gunma Prefectural College of Health Sciences, 323-1 Kamioki-machi, Maebashi-shi 371-0052, Japan. ${ }^{3}$ National Institute of Radiological Sciences, National Institutes for Quantum and Radiological Science and Technology, 4-9-1 Anagawa, Inage-ku, Chiba-shi 263-8555, Japan. ${ }^{4}$ Department of Molecular Imaging, Institute of Biomedical Research and Innovation, 2-2, Minatojima Minamimachi, Chuo-ku, Tokyo 28 650-0047, Japan. ${ }^{5}$ Department of Nuclear Medicine, Cancer Institute Hospital of Japanese Foundation for Cancer Research, 3-8-31 Ariake, Koto-ku, Tokyo 135-8550, Japan. 'Department of Radiology, Yokohama City University, 3-9 Fukuura, Kanazawa-ku, Yokohama 236-0004, Japan. 7 Department of Radiology, Center Hospital of National Center for Global Health and Medicine, 1-21-1 Toyama Shinjuku-ku, Tokyo 162-8655, Japan. ${ }^{8}$ Department of Radiology, Kyoto University Hospital, 54 Kawaharacho, Syogoin, Sakyo-ku, Kyoto City 606-8507, Japan. ${ }^{9} \mathrm{Clinical}$ Imaging Center for Healthcare, Nippon Medical School, 1-12-15 Sendagi, Bunkyo-ku, Tokyo 113-0022, Japan. ${ }^{10}$ Division of Medical Imaging and Technology, Hokkaido University Hospital, Kita 14-jo Nishi 5-chome, Kita-ku, Sapporo-shi, Hokkaido 060-8648, Japan. ${ }^{11}$ Department of Radiology, Toranomon Hospital, 2-2-2 Toranomon, Minato-ku, Tokyo 105-8470, Japan.

${ }^{12}$ Department of Health Science, Faculty of Medical Sciences, Kyushu University, 3-1-1 Maidashi, Higashi-ku, Fukuoka 812-8582, Japan.

Received: 10 April 2018 Accepted: 9 August 2018

Published online: 20 August 2018

References

1. Fletcher JW, Djulbegovic B, Soares HP, Siegel BA, Lowe VJ, Lyman GH, et al. Recommendations on the use of 18F-FDG PET in oncology. J Nucl Med. 2008:49:480-508.

2. Ben-Haim S, Ell P. 18F-FDG PET and PET/CT in the evaluation of cancer treatment response. J Nucl Med. 2009;50:88-99.

3. Gupta T, Master Z, Kannan S, Agarwal JP, Ghsoh-Laskar S, Rangarajan V, et al. Diagnostic performance of post-treatment FDG PET or FDG PET/CT imaging in head and neck cancer: a systematic review and meta-analysis. Eur J Nucl Med Mol Imaging. 2011;38:2083-95.

4. Bengtsson T, Hicks RJ, Peterson A, Port RE. 18F-FDG PET as a surrogate biomarker in non-small cell lung cancer treated with erlotinib: newly identified lesions are more informative than standardized uptake value. J Nucl Med. 2012:53:530-7.

5. Hicks RJ. Role of $18 \mathrm{~F}-F D G$ PET in assessment of response in non-small cell lung cancer. J Nucl Med. 2009;50(Suppl 1):31S-42S.

6. Hellwig D, Graeter TP, Ukena D, Groeschel A, Sybrecht GW, Schaefers HJ, et al. 18F-FDG PET for mediastinal staging of lung cancer: which SUV threshold makes sense? J Nucl Med. 2007:48:1761-6.

7. Dijkman BG, Schuurbiers OCJ, Vriens D, Looijen-Salamon M, Bussink J, Timmer-Bonte $\mathrm{JNH}$, et al. The role of (18)F-FDG PET in the differentiation between lung metastases and synchronous second primary lung tumours. Eur J Nucl Med Mol Imaging. 2010;37:2037-47.

8. Agarwal M, Brahmanday G, Bajaj SK, Ravikrishnan KP, Wong C-YO. Revisiting the prognostic value of preoperative (18)F-fluoro-2-deoxyglucose ( (18)FFDG) positron emission tomography (PET) in early-stage (I \& II) non-small cell lung cancers (NSCLC). Eur J Nucl Med Mol Imaging. 2010;37:691-8.

9. Liao S, Penney BC, Wroblewski K, Zhang H, Simon CA, Kampalath R, et al. Prognostic value of metabolic tumor burden on 18F-FDG PET in nonsurgical patients with non-small cell lung cancer. Eur J Nucl Med Mol Imaging. 2012;39:27-38.

10. Sullivan DC, Obuchowski NA, Kessler LG, Raunig DL, Gatsonis C, Huang EP et al. Metrology standards for quantitative imaging biomarkers. Radiology. 2015;277:813-25.

11. O'Connor JPB, Aboagye EO, Adams JE, Aerts HJWL, Barrington SF, Beer AJ, et al. Imaging biomarker roadmap for cancer studies. Nat Rev Clin Oncol. 2017;14:169-86. Nature Publishing Group 
12. Boellaard R, Krak NC, Hoekstra OS, Lammertsma AA. Effects of noise, image resolution, and $\mathrm{ROI}$ definition on the accuracy of standard uptake values: a simulation study. J Nucl Med. 2004;45:1519-27.

13. Westerterp M, Pruim J, Oyen W, Hoekstra O, Paans A, Visser E, et al. Quantification of FDG PET studies using standardised uptake values in multi-centre trials: effects of image reconstruction, resolution and $\mathrm{ROI}$ definition parameters. Eur J Nucl Med Mol Imaging. 2007;34:392-404.

14. Sunderland JJ, Christian PE. Quantitative PET/CT scanner performance characterization based upon the Society of Nuclear Medicine and Molecular Imaging Clinical Trials Network oncology clinical simulator phantom. J Nucl Med. 2015:56:145-52.

15. Lasnon C, Desmonts C, Quak E, Gervais R, Do P, Dubos-Arvis C, et al. Harmonizing SUVs in multicentre trials when using different generation PET systems: prospective validation in non-small cell lung cancer patients. Eur J Nucl Med Mol Imaging. 2013;40:985-96.

16. Rahmim A, Qi J, Sossi V. Resolution modeling in PET imaging: theory, practice, benefits, and pitfalls. Med Phys. 2013;40:64301.

17. Kidera D, Kihara K, Akamatsu G, Mikasa S, Taniguchi T, Tsutsui Y, et al. The edge artifact in the point-spread function-based PET reconstruction at different sphere-to-background ratios of radioactivity. Ann Nucl Med. 2016; 30:97-103.

18. Graham MM, Wahl RL, Hoffman JM, Yap JT, Sunderland JJ, Boellaard R, et al. Summary of the UPICT protocol for 18F-FDG PET/CT imaging in oncology clinical trials. J Nucl Med. 2015;56:955-61.

19. Boellaard R, Delgado-Bolton R, Oyen WJG, Giammarile F, Tatsch K, Eschner W, et al. FDG PET/CT: EANM procedure guidelines for tumour imaging: version 2.0. Eur J Nucl Med Mol Imaging. 2015;42:328-54.

20. Fukukita H, Suzuki K, Matsumoto K, Terauchi T, Daisaki H, Ikari Y, et al. Japanese guideline for the oncology FDG-PET/CT data acquisition protocol: synopsis of version 2.0. Ann. Nucl Med. 2014;28:693-705.

21. Japanese Society of Nuclear Medicine. Standard PET imaging protocols and phantom test procedures and criteria: executive summary. http://jsnm. sakura.ne.jp/wp_jsnm/wp-content/themes/theme_jsnm/doc/ StandardPETProtocolPhantom20170201.pdf. Accessed 1 Dec 2017.

22. Joshi A, Koeppe RA, Fessler JA. Reducing between scanner differences in multi-center PET studies. Neurolmage. 2009:46(1):154-9.

23. Quak E, Le Roux P-Y, Hofman MS, Robin P, Bourhis D, Callahan J, et al. Harmonizing FDG PET quantification while maintaining optimal lesion detection: prospective multicentre validation in 517 oncology patients. Eur J Nucl Med Mol Imaging. 2015;42:2072-82.

24. Makris NE, Huisman MC, Kinahan PE, Lammertsma AA, Boellaard R. Evaluation of strategies towards harmonization of FDG PET/CT studies in multicentre trials: comparison of scanner validation phantoms and data analysis procedures. Eur J Nucl Med Mol Imaging. 2013;40:1507-15.

25. Quak E, Le Roux P-Y, Lasnon C, Robin P, Hofman MS, Bourhis D, et al. Does PET SUV harmonization affect PERCIST response classification? J Nucl Med. 2016:57:1699-706.

26. Raunig DL, McShane LM, Pennello G, Gatsonis C, Carson PL, Voyvodic JT, et al. Quantitative imaging biomarkers: a review of statistical methods for technical performance assessment. Stat Methods Med Res. 2015;24:27-67.

27. Pierce LA, Elston BF, Clunie DA, Nelson D, Kinahan PE. A digital reference object to analyze calculation accuracy of PET standardized uptake value. Radiology. 2015;277:538-45.

28. Quantitative imaging biomarker alliance. QIBA Profile. FDG-PET/CT for Response to Cancer Therapy. Available at: URL: https://qibawiki.rsna.org/ images/1/1f/QIBA_FDG-PET_Profile_v113.pdf. Accessed 28 July 2017.

29. Nakahara T, Daisaki H, Yamamoto $Y$, limori T, Miyagawa K, Okamoto T, et al. Use of a digital phantom developed by QIBA for harmonizing SUVS obtained from the state-of-the-art SPECT/CT systems: a multicenter study. EJNMMI Res. 2017;7:53.

30. Boellaard R. Standards for PET image acquisition and quantitative data analysis. J Nucl Med. 2009;50(Suppl 1):11S-20S.

31. Kelly MD, Declerck JM. SUVref: reducing reconstruction-dependent variation in PET SUV. EJNMMI Res. 2011;1:16. Springer Open Ltd

32. Takahashi Y, Oriuchi N, Otake H, Endo K, Murase K. Variability of lesion detectability and standardized uptake value according to the acquisition procedure and reconstruction among five PET scanners. Ann Nucl Med. 2008:22:543-8.

33. Velasquez LM, Boellaard R, Kollia G, Hayes W, Hoekstra OS, Lammertsma AA, et al. Repeatability of 18F-FDG PET in a multicenter phase I study of patients with advanced gastrointestinal malignancies. J Nucl Med. 2009;50:1646-54.
34. Doot RK, Scheuermann JS, Christian PE, Karp JS, Kinahan PE. Instrumentation factors affecting variance and bias of quantifying tracer uptake with PET/CT. Med Phys. 2010;37:6035-46.

35. Lodge MA. Repeatability of SUV in oncologic (18)F-FDG PET. J Nucl Med. 2017;58:523-32

36. Lim R, Eaton A, Lee NY, Setton J, Ohri N, Rao S, et al. 18F-FDG PET/CT metabolic tumor volume and total lesion glycolysis predict outcome in oropharyngeal squamous cell carcinoma. J Nucl Med. 2012;53:1506-13.

\section{Submit your manuscript to a SpringerOpen ${ }^{\circ}$ journal and benefit from:}

- Convenient online submission

- Rigorous peer review

- Open access: articles freely available online

- High visibility within the field

- Retaining the copyright to your article

Submit your next manuscript at $\boldsymbol{\nabla}$ springeropen.com 